\title{
Deciphering the link between salicylic acid signaling and sphingolipid metabolism
}

\author{
Diana Sánchez-Rangel ${ }^{\dagger}$, Mariana Rivas-San Vicente, \\ M. Eugenia de la Torre-Hernández, Manuela Nájera-Martínez and Javier Plasencia *
}

Departamento de Bioquímica, Facultad de Química, Universidad Nacional Autónoma de México, México City, México

\section{OPEN ACCESS}

Edited by:

Hua Lu, University of Maryland, Baltimore County, USA

Reviewed by:

Shunyuan Xiao, University of Maryland, USA

Robin Katrina Cameron, McMaster University, Canada

*Correspondence: Javier Plasencia,

Departamento de Bioquímica, Facultad de Química, Universidad Nacional Autónoma de México, 04510 México City, México javierp@unam.mx

${ }^{\dagger}$ Present address: Investigador Cátedras CONACYT adscrita al Instituto de Ecología A. C.,

Red de Estudios Moleculares

Avanzados Carretera Antigua a

Coatepec 351, El Haya, 91070

Xalapa, Veracruz, México

Specialty section:

This article was submitted to Plant-Microbe Interaction, a section of the journal Frontiers in Plant Science

Received: 14 October 2014

Accepted: 16 February 2015

Published: 09 March 2015

Citation:

Sánchez-Rangel D, Rivas-San Vicente M, de la Torre-Hernández ME, Nájera-Martínez M and Plasencia J (2015) Deciphering the link between salicylic acid signaling and sphingolipid metabolism.

Front. Plant Sci. 6:125.

doi: 10.3389/fpls.2015.00125
The field of plant sphingolipid biology has evolved in recent years. Sphingolipids are abundant in cell membranes, and genetic analyses revealed essential roles for these lipids in plant growth, development, and responses to abiotic and biotic stress. Salicylic acid (SA) is a key signaling molecule that is required for induction of defense-related genes and rapid and localized cell death at the site of pathogen infection (hypersensitive response) during incompatible host-pathogen interactions. Conceivably, while levels of SA rapidly increase upon pathogen infection for defense activation, they must be tightly regulated during plant growth and development in the absence of pathogens. Genetic and biochemical evidence suggest that the sphingolipid intermediates, longchain sphingoid bases, and ceramides, play a role in regulating SA accumulation in plant cells. However, how signals generated from the perturbation of these key sphingolipid intermediates are transduced into the activation of the SA pathway has long remained to be an interesting open question. At least four types of molecules - MAP kinase 6, reactive oxygen species, free calcium, and nitric oxide - could constitute a mechanistic link between sphingolipid metabolism and SA accumulation and signaling.

Keywords: salicylic acid, sphingolipid, ceramide, sphingoid bases, sphinganine-analog mycotoxin

\section{Introduction}

Salicylic acid (SA) is a phytohormone involved in local and systemic resistance (Vlot et al., 2009), as well as in the response to abiotic stress, growth, and development (Rivas-San Vicente and Plasencia, 2011). The SA signaling pathway requires a functional NPR1 [nonexpressor of pathogenesis-related (PR) genes 1] protein to relay the signal to the nucleus, where it activates PR gene expression (Wang et al., 2005; Kumar, 2014; Seyfferth and Tsuda, 2014). SA biosynthesis occurs either through the phenylalanine (PAL) or isochorismate (ICS) pathway, and the relative contribution of each route varies in different species (Chen et al., 2009b; An and Mou, 2011). SA production is controlled by multiple positive and negative regulators (Janda and Ruelland, 2014). Exciting new research reveals that several sphingolipid intermediates induce SA accumulation and affect disease resistance. The objective of this review is to assess the experimental data that link sphingolipid metabolism with SA accumulation and signaling. Such evidence is mainly derived from (1) the phenotypes of Arabidopsis and Nicotiana plants in which genes involved in sphingolipid metabolism are mutated or silenced, and (2) the effects of sphinganine analog mycotoxins (SAMs, namely AAL and FB1) on sphingolipid metabolism. 


\section{Sphingolipid Metabolism}

Research in plant sphingolipids has been fostered by the use of novel extraction protocols, followed by mass spectrometry analysis and characterization of Arabidopsis mutants. Sphingolipids compose $\sim 40 \%$ of the lipids of the plasma membrane and are also abundant in other endomembranes. Functional genomics of sphingolipid metabolism genes show that these molecules have essential functions in plant growth, development, and stress responses (Chen et al., 2009a; Pata et al., 2010; Berkey et al., 2012). Sphingolipid biosynthesis starts in the endoplasmic reticulum (ER). L-serine is condensed with palmitoyl-CoA to generate a sphingoid long-chain base (LCB) that is reduced and then $\mathrm{N}$-acylated to form ceramide. Ceramides are substrates for the production of complex sphingolipids, including inositol phosphorylceramide (IPC), and glucosylceramide. In addition to hydroxylation, LCBs and ceramides can be phosphorylated (Figure 1) to yield a wide variety of molecules (Markham et al., 2006).

\section{Disruption of Sphingolipid Metabolism Through Mutation and Silencing Affects Salicylic Acid Levels}

In Table 1, we summarize the effects of mutation or silencing of genes involved in sphingolipid biosynthesis or metabolism in Arabidopsis ecotypes and Nicotiana benthamiana, and highlights the effects of altered SA levels and/or signaling on PR1 gene expression.

\section{Sphingolipid Biosynthesis and Metabolism Long-Chain Base Biosynthesis}

Serine palmitoyl transferase (SPT), a heterodimer formed by LCB1 and LCB2 subunits, catalyzes the first reaction in sphingolipid biosynthesis to form LCBs (Figure 1; Chen et al., 2009a). The Arabidopsis genome contains one gene encoding the LCB1 subunit and two encoding LCB2. Functional studies using mutant and RNAi suppression lines lacking LCB1 expression, and double mutants lacking both LCB2 genes, show that sphingolipids are essential for growth and development (Chen et al., 2006; Dietrich et al., 2008). However, it is unknown whether mutations in any of the $L C B$ genes affect the SA pathway. A link between SA and sphingolipid metabolism was established through virusinduced gene silencing (VIGS) of the N. benthamiana LCB2 subunit. A 20 to 50\% reduction in NbLCB2 transcript level was sufficient to impair growth and leaf and flower development. Compared to control plants, plants with a $\sim 50 \%$ reduction in $\mathrm{NbLCB} 2$ transcripts display elevated SA levels and constitutive PR1 expression (Table 1), and later, show spontaneous cell death in leaves. These silenced plants are also more susceptible to infection by the fungal necrotroph Alternaria alternata f. sp. lycopersici. LCB composition in silenced plants is altered with lower trihydroxylated LCB and higher dihydroxylated LCB levels than those of control plants (Rivas-San Vicente et al., 2013). These results suggest that disruption of LCB homeostasis is accompanied by elevated SA levels and induction of cell death.
However, the identity of the LCB responsible for this phenotype is unknown.

\section{Ceramide Biosynthesis}

Ceramide synthase catalyzes the condensation of a LCB with a fatty acid-CoA to yield ceramide (Figure 1). The Arabidopsis genome has three ceramide synthase genes $-\mathrm{LOH} 1, \mathrm{LOH} 2$, and $\mathrm{LOH} 3$ - and each isoform has a selective preference for the type of acyl-CoA and LCB (dihydroxy- or trihydroxy-LCB; Chen et al., 2009a). Mutants for each gene are viable, and only the loh1 line has a spontaneous cell death phenotype, which occurs late in development. Although SA levels in this mutant are comparable to those in wild type (WT) plants, PR1 transcription increased 160-fold (Table 1). Furthermore, this mutant exhibits modest changes in sphingolipid content, with a 7 and $19 \%$ increase in the proportion of species containing a C16 fatty acid in ceramides and GlcCer, respectively, and a fivefold increase in trihydroxyLCBs (Ternes et al., 2011). These data narrow-down the identity of the bioactive sphingolipids responsible for triggering cell death to free trihydroxy-LCBs, dihydroxy-LCBs, or ceramide species with a C16 fatty acid.

\section{Ceramide Hydroxylation}

Ceramide might be hydroxylated in its LCB moiety by a LCB$\mathrm{C} 4$ hydroxylases $(\mathrm{SBH})$ and/or at the fatty acid residues by fatty acid hydroxylases (FAH; Markham et al., 2006). Although double mutants and RNAi suppression lines of $S B H$ genes display necrotic lesions in their cotyledons, and constitutively express $P R$ genes (Chen et al., 2008), data on SA accumulation and/or signaling is lacking. Conversely, an analysis of double mutants of the two FAH genes ( $f a h 1$ and fah2) demonstrated a link between sphingolipid biosynthesis and SA metabolism. The fah1/fah 2 double mutant displays a $25 \%$ reduction in leaf and root growth compared to WT plants, elevated SA levels, and aberrant constitutive PR1 expression (Table 1). Despite elevated SA levels, this mutant lacks a spontaneous cell death phenotype. These plants contain lower levels of ceramides and GlcCer with $\alpha$-hydroxylated fatty acids, but a 10-fold increase in phytoceramides and a fivefold increase in trihydroxylated LCBs (König et al., 2012). Thus fatty acid hydroxylation of ceramides is required for the biosynthesis of complex sphingolipids and its absence leads to the accumulation of LCBs and ceramides. This elevation activates the SA pathway and supports a link between SA signaling and sphingolipid metabolism.

\section{Ceramide Phosphorylation}

ACD5 encodes a 608-amino acid protein with ceramide kinase activity that is located in the ER, Golgi apparatus (GA), and mitochondria (Liang et al., 2003; Bi et al., 2014). In the Arabidopsis acd5 mutant, a glycine residue is replaced with an arginine and the mutant enzyme retains only $10 \%$ of the activity of the WT. These mutant plants develop normally for 5 weeks and then display spontaneous leaf lesions, accumulate free, and conjugated SA along with reactive oxygen species (ROS), and constitutively express PR1 (Table 1). Due to reduced ceramide kinase activity, acd5 plants accumulate ceramides and hydroxyceramides, with a two- to sixfold increase relative to WT plants. Only ceramides 


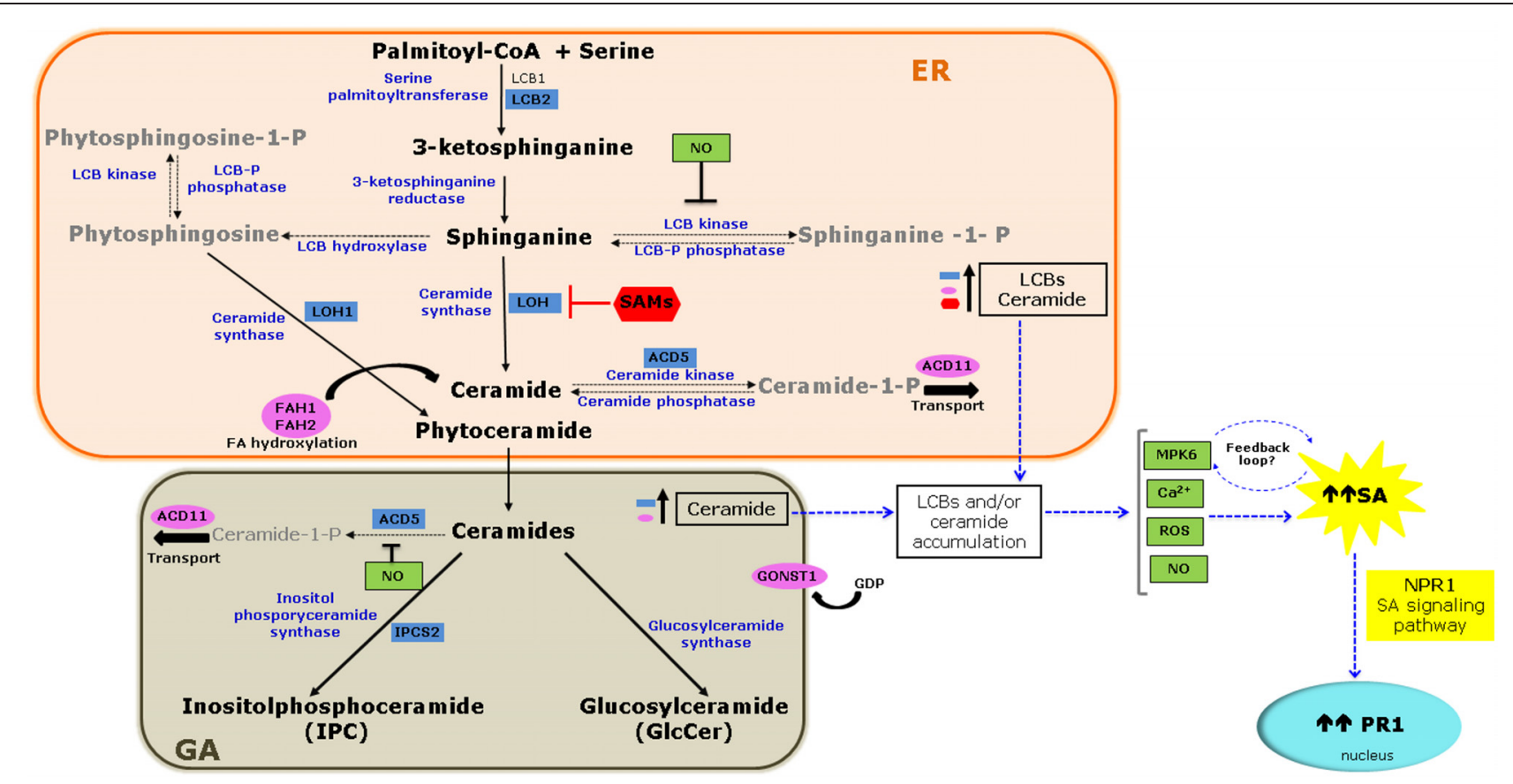

FIGURE 1 | Molecular links between sphingolipid metabolism and the salicylic acid (SA) signaling pathway. De novo biosynthesis of sphingolipids starts in the endoplasmic reticulum (ER) and ends in the Golgi apparatus (GA) with the biosynthesis of complex sphingolipids. The diagram highlights mutations or silencing events in Arabidopsis and Nicotiana that result in loss or reduced function of genes involved in sphingolipid metabolism (blue boxes) or sphingolipid modification or transport (pink ovals) and their effect on SA accumulation and/or signaling as a consequence of the accumulation of long-chain base (LCBs), and/or ceramide (see Table 1). Also illustrated is the inhibition of the ceramide synthase by sphinganine analog mycotoxin (SAMs; red) that additionally contributes to LCB accumulation and SA pathway activation through pathogenesis-related (PR1) gene 1 expression. Several signaling molecules are candidates connecting sphingolipid metabolism and SA signaling (green boxes). MITOGEN-ACTIVATED PROTEIN KINASE 6 (MPK6) may act in a feedback loop between the two pathways. Solid arrows indicate biosynthetic steps, while dashed arrows indicate modification of LCBs and ceramide. Blue arrows denote proposed steps of convergence between the two pathways. containing long-chain fatty acids (C16) accumulate while levels of ceramides with very-long-chain fatty acids (C24 and C26) are not altered (Bi et al., 2014).

The acd5 cell death phenotype is SA-dependent as it is suppressed in the acd5/NahG genotype. NahG encodes a salicylate hydroxylase which converts SA to inert catechol such that these plants do not accumulate SA. Moreover, a functional SA signaling pathway is required because acd5/npr1 double mutants have an attenuated cell death phenotype (Greenberg et al., 2000; Liang et al., 2003; Bi et al., 2014). Mutation in the ACD5 gene causes an imbalance in the ceramide to ceramide-1-phosphate ratio and ceramide accumulation might activate the SA pathway.

\section{Inositol Phosphorylceramide Biosynthesis}

Ceramides serve as substrates for the formation of complex sphingolipids (Figure 1). Inositol phosphorylceramide-synthase (IPCS) catalyzes the transfer of phosphorylinositol to phytoceramide to yield IPC. The Arabidopsis genome contains three functional IPCS genes: IPCS1, IPCS2, and IPCS3. AtIPCS2 is expressed at higher levels than the other two genes in all organs tested (Mina et al., 2010), and the protein localizes to the transGolgi network (Wang et al., 2008).

The phenotype associated with IPCS2 loss of function is only discernible in transgenic plants expressing the resistance gene
RPW8 ( Resistance to powdery mildew). These plants exhibit spontaneous cell death and were thus named ehr1 (enhancing $\underline{R} P W 8$ mediated hypersensitive response cell death). Arabidopsis RPW8 confers broad-spectrum resistance to powdery mildew, and the ipcs 2 mutation in $R P W 8$ transgenic plants increased sensitivity to fungal infection. The ipcs 2 mutant lines are only $30 \%$ the size of the parental line at maturity and exhibit regions of spontaneous cell death. These plants accumulate both free SA and SA conjugates and constitutively express $P R 1$ (Table 1). Spontaneous cell death and constitutive PR1 expression depend on SA, since both traits are abolished when the NahG gene or the pad4 mutation is introduced. PAD4 is an upstream regulator of the SA signaling pathway. Again, the precise identity of the sphingolipid molecule responsible for this phenotype remains unidentified, because these mutant plants show increased levels of both ceramides and LCBs (Wang et al., 2008).

\section{Sphingolipid Modification}

Complex sphingolipids, such as glycosyl IPC (GIPC), are the most abundant lipids in plant cell membranes (Markham et al., 2006). Glycosylation of the inositol head group occurs in the GA. Monosaccharides such as hexuronic acid, galactose, mannose, and arabinose can be attached to GIPC (Buré et al., 2011). Guanidine diphosphate (GDP) sugars serve 


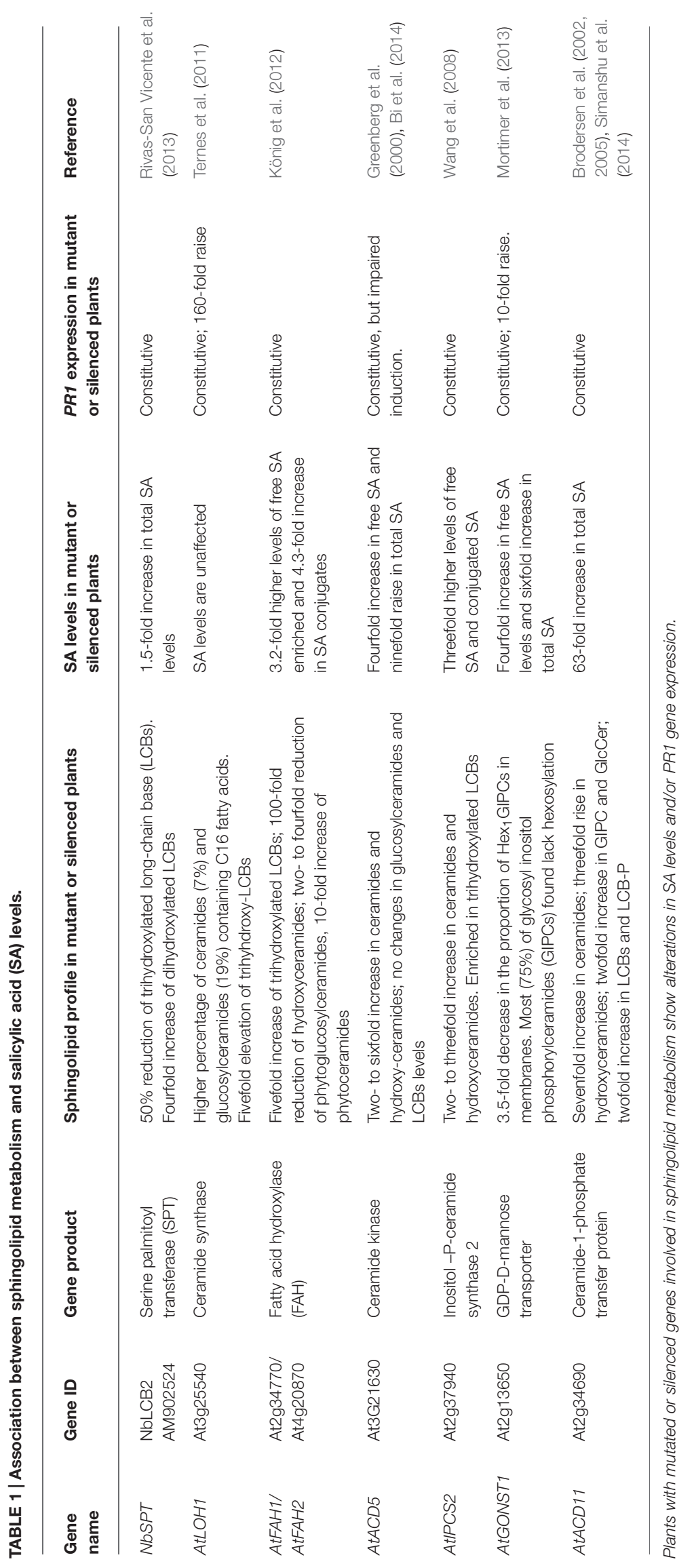


as donors for glycosylation and are transported into the GA. GONST1 (GOLGI-LOCALIZED NUCLEOTIDE SUGAR TRANSPORTER) belongs to a family of nucleotide sugar transporters and stimulates GDP-mannose transport (Baldwin et al., 2001). Knock-out of GONST1 causes severe dwarfing, poor seed set, formation of spontaneous necrotic lesions on the leaves, accumulation of free and conjugated SA, and constitutive PR1 expression (Table 1). Overexpression of Nah G in the gonst 1 background diminishes SA levels and the number of necrotic lesions, and partially alleviates the growth defect. These data suggest that the ability to accumulate SA is partly responsible for the gonst1 phenotype. The gonst 1 plants do not differ from WT plants in ceramide or LCB content, but do exhibit changes in sphingolipid sugar decoration; the proportion of $\mathrm{Hex}_{1}$ GIPCs in membranes isolated from gonst 1 is $25 \%$, compared to $90 \%$ in the WT (Mortimer et al., 2013).

\section{Sphingolipid Transport}

$A C D 11$ encodes a protein homologous to a mammalian glycolipid transfer protein (GLTP) with no predicted transmembrane domains or localization motifs. It was initially characterized as a sphingosine transporter (Brodersen et al., 2002), but a recent study showed that this protein contains a lipid recognition center. ACD11 selectively binds to ceramide-1-phosphate (C1P) and phyto-C1P, but not to related plant sphingolipids such as ceramides, GlcCers, GIPCs, and LCBs (Simanshu et al., 2014).

Arabidopsis acd11 mutant plants show an accelerated cell death phenotype early in development, characterized by ROS generation, necrotic lesions, and constitutive expression of senescence- and defense-related genes (Brodersen et al., 2002). They also accumulate SA and display constitutive PR1 expression (Table 1). Since the cell death phenotype is suppressed in transgenic NahG lines that do not accumulate SA, cell death is SA-dependent. Cell death is also blocked by mutations in PAD4 and EDS1, which are upstream regulators of the SA response (Brodersen et al., 2002). Other mutations in key components of the SA biosynthesis and signaling pathway, such as SID2 and EDS5, also diminish SA accumulation in the acd11 mutant (Brodersen et al., 2005). SID2 encodes an ICS synthase, suggesting that SA accumulation is partly responsible for the observed phenotype, and ICS precursors might trigger cell death. The acd11/eds5 mutant exhibits constitutive PR1 expression and a similar cell death phenotype as the acd11 mutants, but limited SA accumulation. EDS5 encodes an extrusion-like transporter involved in SA export from chloroplasts (Serrano et al., 2013). Exogenous application of the SA ana$\log$ BTH to acd11/NahG and acd11/sid2 plants restores cell death and induces ceramide accumulation, reinforcing SA role in the signaling pathway that results in this phenotype (Brodersen et al., 2002, 2005).

Both LCBs and ceramides could contribute to the acd11 mutant phenotype, as levels of these sphingolipid intermediates are elevated in these plants. Because of the functional association between sphingolipid metabolism and SA biosynthesis, a feedback loop might regulate these two pathways (Simanshu et al., 2014).

\section{SA Levels are Affected by the Action of Sphinganine Analog Mycotoxins on Sphingolipid Metabolism}

SAMs share structural similarity with LCBs and inhibit ceramide synthase activity (Figure 1) causing LCB levels to increase (Wang et al., 1991; Abbas et al., 1994). The best-characterized SAMs are the AAL-toxin produced by A. alternata f. sp. lycopersici, a tomato foliar pathogen, and fumonisin B1 (FB1), produced by Fusarium verticillioides, a causal agent of various diseases in maize. Although the hosts and type of diseases caused by these fungi are quite different, genetic evidence supports a role for SAMs in virulence of these fungal pathogens (Sánchez-Rangel and Plasencia, 2010).

\section{Effects of SAMs in Arabidopsis Genotypes and Hormonal Crosstalk}

Fumonisin B1 causes LCB accumulation in several plant species (Abbas et al., 1994; de la Torre-Hernandez et al., 2010), and in Arabidopsis, a 72-h treatment with $10 \mu \mathrm{M} \mathrm{FB} 1$ triggers a 100 - to 7000-fold increase in LCBs concentration (Saucedo-García et al., 2011). This dose also triggers lesions reminiscent of those formed in the pathogen-induced HR, accompanied by callose deposition, ROS and camalexin production, and expression of $P R-1$, $P R-2$, and $P R-5$ in Arabidopsis leaves (Stone et al., 2000). Very low doses $(70 \mathrm{nM})$ of FB1 cause DNA fragmentation and cell death in Arabidopsis protoplasts. Because protoplasts from mutant genotypes defective in SA, jasmonic acid (JA), and ethylene (ET) accumulation or signaling are more tolerant to the toxin, it was concluded that all three signaling pathways are required for cell death caused by FB1 (Asai et al., 2000). However, when $50 \mu \mathrm{M}$ FB1 was infiltrated into rosette leaves of $d d e 2$, ein2, pad4, and sid2 single mutants (defective in JA, ET, SA, and SA signaling pathways, respectively) and the corresponding quadruple mutant, enhanced tolerance to FB1 was not observed (Igarashi et al., 2013). The cell type tested - protoplasts vs. intact leaves - and the $\sim 700$-fold difference in FB1 dose might account for the observed discrepancies.

From the above data, it is clear that FB1 activates the SA pathway, but also other routes that are antagonistic to SA signaling, thus complicating results interpretation. For instance, Arabidopsis possesses five ET receptors (ETR1, ETR2, EIN4, ERS1, and ERS2) and genetic studies show that, in absence of ET, the receptors positively regulate CTR1, which acts as a negative regulator of the ET signaling pathway (Ju et al., 2012). FB1 has contrasting effects in mutants of the five ET receptors; while the etr1-1 mutant shows hypersensitivity to FB1, the ein4-1 mutant displays diminished cell death and the other three mutants respond similarly to the WT. These results suggest that ET receptors have distinct roles in toxin sensitivity leading to the HR. While ET induces cell death through EIN4, perception of this phytohormone by ETR1 inhibits cell death. Because ET represses PR1 transcription, mutations in genes encoding ET receptors increase the expression of SA-inducible genes; for instance the ers 1 and the ein 4 mutants display a 29 -fold and 115-fold rise in PR1 expression, respectively (Plett et al., 2009). 


\section{Deciphering the Link Between Sphingolipid Metabolism and the SA Pathway}

Since both, LCBs and ceramides, serve as signaling molecules in the activation of defense-related PCD (Berkey et al., 2012), it is reasonable to hypothesize that SA acts as an intermediate in this pathway. So far, evidence provided by mutants in sphingolipid biosynthesis and by experiments with SAMs and exogenous LCBs/ceramides, suggests that sphingolipid intermediates act upstream of SA. Expression of NahG or negative regulators of the SA pathway in sphingolipid mutants confirms that this phytohormone is required for the cell death phenotype. However, the remaining question is how perturbations in levels of LCBs/ceramides are perceived to induce the SA biosynthesis pathway? Several signaling molecules, upstream and/or downstream of sphingolipid intermediates, are candidates to connect these two pathways (Figure 1), will be described briefly.

Both, FB1 and LCBs activate MITOGEN-ACTIVATED PROTEIN KINASE 6 (MPK6) within minutes after the infiltration of Arabidopsis rosette leaves. Moreover, mpk6 mutant seedlings show reduced cell death when exposed to $10 \mu \mathrm{M}$ FB1, suggesting that MPK6 is a transducer in the pathway leading to LCB-induced PCD in Arabidopsis (Saucedo-García et al., 2011). Although MPK6 was characterized as the ortholog of the tobacco SA-induced protein kinase (SIPK), it is rapidly activated by several microbial elicitors (Nühse et al., 2000) with a similar kinetics as with FB1 and LCBs.

Reactive oxygen species elevation is a common feature displayed by several mutants in sphingolipid biosynthesis that show an enhanced cell death phenotype and SA accumulation. Molecules such as hydrogen peroxide $\left(\mathrm{H}_{2} \mathrm{O}_{2}\right)$ and superoxide anion $\left(\mathrm{O}_{2}^{-}\right)$mediate a variety of cellular responses. In Arabidopsis, $10 \mu \mathrm{M} \mathrm{FB1}$ causes an elevation of $P A L$ transcript and activity, which results in a fourfold increase in total SA. This elevation depends on ROS, as inhibitors that disrupt ROS production prevent this response (Xing et al., 2013). Moreover, exogenous LCBs induce ROS production in leaves of Arabidopsis seedlings (Shi et al., 2007). Although data on LCB accumulation is lacking in these reports, FB1 biological activity suggests that LCBs elevation mediate ROS generation.

Another hypothesis is that free calcium levels change in response to a sphingolipid imbalance and transduce a signal. In tobacco BY2 cells, exogenous addition of dihydroxyLCB causes an immediate $(\sim 1 \mathrm{~min})$ dose-dependent elevation of cellular free calcium concentration in the cytosol

\section{References}

Abbas, H. K., Tanaka, T., Duke, S. O., Porter, J. K., Wray, E. M., Hodges, L., et al. (1994). Fumonisin- and AAL-toxin-induced disruption of sphingolipid metabolism with accumulation of free sphingoid bases. Plant Physiol. 106, 1085-1093.

An, C., and Mou, Z. (2011). Salicylic acid and its function in plant immunity. J. Int. Plant Biol. 53, 412-428. doi: 10.1111/j.1744-7909.2011.01043.x

Asai, T., Stone, J. M., Heard, J. E., Kovtun, Y., Yorgey, P., Sheen, J., et al. (2000). Fumonisin B1-induced cell death in Arabidopsis protoplasts requires and 10 min later in the nucleus, followed by $\mathrm{H}_{2} \mathrm{O}_{2}$ accumulation and cell death (Lachaud et al., 2010, 2011). Calcium also regulates the expression of the SA biosynthesis gene ICS1 through the $\mathrm{Ca}^{2+} /$ calmodulin-binding transcription factor CBP60g (Wang et al., 2009; Zhang et al., 2010). Another $\mathrm{Ca}^{2+} /$ calmodulin-binding transcription factor, AtSR1, is a negative regulator of SA signaling, as it controls EDS1 expression (Du et al., 2009). Because calcium controls SA levels, free cytosolic $\mathrm{Ca}^{2+}$ could link sphingolipid metabolism with the SA pathway. Testing the susceptibility of atsr1 mutants when challenged to an imbalance of sphingolipids levels through exogenous addition of FB1 or LCBs might shed some light on this question.

Finally, nitric oxide (NO), a universal transducer molecule, might play a role in linking sphingolipids and the SA pathway. In Taxus sp. cell cultures, a fungal-produced sphingolipid induces rapid and dose-dependent NO production, and because this molecule is a redox regulator of the NPR1/TGA1 system, it promotes NPR1 translocation into the nucleus (Wang et al., 2007; Lindermayr et al., 2010; Guillas et al., 2013). NO might also act upstream of sphingolipids intermediates. Exposure of Arabidopsis plants to cold induce NO production that downregulates the synthesis of phytosphingosine-phosphate and ceramide-phosphate. In the nia1/nia2 nitrate reductase mutant, impaired in NO biosynthesis, such suppression does not occur (Cantrel et al., 2011). Thus NO could participate in the finetuning of the balance between certain sphingolipids and their phosphorylated derivatives.

Studies of the phenotypes of Arabidopsis mutants in sphingolipid metabolism suggest that imbalance of LCBs and/or ceramides levels activate the SA pathway. However, further research is needed to determine the causality of this relationship and to identify the upstream signal transduction molecule(s) responsible for activating the SA pathway. Additional comparisons of the effects of FB1, LCBs, and ceramides on MPK, ROS, calcium, and NO signaling in relevant Arabidopsis WT and mutants will reveal the main players in this complex interaction between the sphingolipid and SA signaling pathways.

\section{Acknowledgments}

The authors acknowledge both reviewers for their thorough evaluation of the manuscript and valuable suggestions. Research in JP laboratory is supported by grants from DGAPA-PAPIIT (IN220010) and Programa MASAGRO. DS-R is supported by Cátedras CONACYT program.

jasmonate-, ethylene-, and salicylate-dependent signaling pathways. Plant Cell 12, 1823-1835. doi: 10.1105/tpc.12.10.1823

Baldwin, T. C., Handford, M. G., Yuseff, M. I., Orellana, A., and Dupree, P. (2001). Identification and characterization of GONST1, a golgi-localized GDP-mannose transporter in Arabidopsis. Plant Cell 13, 2283-2295. doi: 10.1105/tpc.13.1 0.2283

Berkey, R., Bendigeri, D., and Xiao, S. (2012). Sphingolipids and plant defense/disease: the "death" connection and beyond. Front. Plant Sci. 3:68. doi: $10.3389 /$ fpls.2012.00068 
Bi, F. C., Liu, Z., Wu, J. X., Liang, H., Xi, X. L., Sun, T. J., et al. (2014). Loss of ceramide kinase in Arabidopsis impairs defenses and promotes ceramide accumulation and mitochondrial $\mathrm{H}_{2} \mathrm{O}_{2}$ bursts. Plant Cell 26, 3449-3467. doi: 10.1105/tpc.114.127050

Brodersen, P., Malinovsky, F. G., Hématy, K., Newman, M. A., and Mundy, J. (2005). The role of salicylic acid in the induction of cell death in Arabidopsis acd11. Plant Physiol. 138, 1037-1045. doi: 10.1104/pp.105.059303

Brodersen, P., Petersen, M., Pike, H. M., Olszak, B., Skov, S., Ødum, N., et al. (2002). Knockout of Arabidopsis accelerated-cell-death11 encoding a sphingosine transfer protein causes activation of programmed cell death and defense. Genes Dev. 16, 490-502. doi: 10.1101/gad.218202

Buré, C., Cacas, J. L., Wang, F., Gaudin, K., Domergue, F., Mongrand, S., et al. (2011). Fast screening of highly glycosylated plant sphingolipids by tandem mass spectrometry. Rapid Commun. Mass Spectrom. 25, 3131-3145. doi: $10.1002 / \mathrm{rcm} .5206$

Cantrel, C., Vazquez, T., Puyaubert, J., Reze, N., Lesch, M., Kaiser, W. M., et al. (2011). Nitric oxide participates in cold-responsive phosphosphingolipid formation and gene expression in Arabidopsis thaliana. New Phytol. 189, 415-427. doi: 10.1111/j.1469-8137.2010.03500.x

Chen, M., Cahoon, E. B., Saucedo-García, M., Plasencia, J., and Gavilanes-Ruíz, M. (2009a). "Plant sphingolipids: structure, synthesis and function," in Lipids in Photosynthesis: Essential and Regulatory Functions, eds H. Wada and N. Murata (Dordrecht: Springer), 77-115.

Chen, Z., Zheng, Z., Huang, J., Lai, Z., and Fan, B. (2009b). Biosynthesis of salicylic acid in plants. Plant Signal. Behav. 4, 493-496. doi: 10.4161/psb.4.6.8392

Chen, M., Han, G., Dietrich, C. R., Dunn, T. M., and Cahoon, E. B. (2006). The essential nature of sphingolipids in plants as revealed by the functional identification and characterization of the Arabidopsis LCB1 subunit of serine palmitoyltransferase. Plant Cell 12, 3576-3593. doi: 10.1105/tpc.105. 040774

Chen, M., Markham, J. E., Dietrich, C. R., Jaworski, J. G., and Cahoon, E. (2008). Sphingolipid long-chain base hydroxylation is important for growth and regulation of sphingolipid content and composition in Arabibopsis. Plant Cell 20, 1862-1878. doi: 10.1105/tpc.107.057851

de la Torre-Hernandez, M. E., Rivas-San Vicente, M., Greaves-Fernandez, N., Cruz-Ortega, R., and Plasencia, J. (2010). Fumonisin B1 induces nuclease activation and salicylic acid accumulation through long-chain sphingoid base build-up in germinating maize. Physiol. Mol. Plant Pathol. 74, 337-345. doi: 10.1016/j.pmpp.2010.05.004

Dietrich, C. R., Han, G., Chen, M., Berg, R. H., Dunn, T. M., and Cahoon, E. B. (2008). Loss-of-function mutations and inducible RNAi suppression of Arabidopsis LCB2 genes reveal the critical role of sphingolipids in gametophytic and sporophytic cell viability. Plant J. 54, 284-298. doi: 10.1111/j.1365313X.2008.03420.x

Du, L., Ali, G. S., Simons, K. A., Hou, J., Yang, T., Reddy, A. S., et al. (2009). $\mathrm{Ca}^{2+} /$ calmodulin regulates salicylic-acid-mediated plant immunity. Nature 457, 1154-1159. doi: 10.1038/nature07612

Greenberg, J. T., Silverman, F. P., and Liang, H. (2000). Uncoupling salicylic aciddependent cell death and defense-related responses from disease resistance in the Arabidopsis mutant acd5. Genetics 156, 341-350.

Guillas, I., Puyaubert, J., and Baudouin, E. (2013). Nitric oxide-sphingolipid interplays in plant signaling: a new enigma from the Sphinx? Front. Plant Sci. 4:341. doi: 10.3389/fpls.2013.00341

Igarashi, D., Bethke, G., Xu, Y., Tsuda, K., Glazebrook, J., and Katagiri, F. (2013). Pattern-triggered immunity suppresses programmed cell death triggered by Fumonisin B1. PLoS ONE 8:e60769. doi: 10.1371/journal.pone.0060769

Janda, M., and Ruelland, E. (2014). Magical mystery tour: salicylic acid signaling. Environ. Exp. Bot. doi: 10.1016/j.envexpbot.2014.07.003

Ju, C., Yoon, G. M., Shemansky, J. M., Lin, D. Y., Ying, Z. I., Chang, J., et al. (2012). CTR1 phosphorylates the central regulator EIN2 to control ethylene hormone signaling from the ER membrane to the nucleus in Arabidopsis. Proc. Natl. Acad. Sci. U.S.A. 109, 19486-19491. doi: 10.1073/pnas.1214848109

König, S., Feussner, K., Schwarz, M., Kaever, A., Iven, T., Landesfeind, M., et al. (2012). Arabidopsis mutants of sphingolipid fatty acid $\alpha$-hydroxylases accumulate ceramides and salicylates. New Phytol. 196, 1086-1097. doi: 10.1111/j.14698137.2012.04351.x

Kumar, D. (2014). Salicylic acid signaling in disease resistance. Plant Sci. 228, 127-124. doi: 10.1016/j.plantsci.2014.04.014
Lachaud, C., Da Silva, D., Amelot, N., Béziat, C., Bière, C., Cotelle, V., et al. (2011). Dihydrosphingosine-induced programmed cell death in tobacco BY2 cells is independent of $\mathrm{H}_{2} \mathrm{O}_{2}$ production. Mol. Plant 4, 310-318. doi: $10.1093 / \mathrm{mp} / \mathrm{ssq} 077$

Lachaud, C., Da Silva, D., Cotelle, V., Thuleau, P., Xiong, T. C., Jauneau, A., et al. (2010). Nuclear calcium controls the apoptotic-like cell death induced by D-erythro-sphinganine in tobacco cells. Cell Calcium 47, 92-100. doi: 10.1016/j.ceca.2009.11.011

Liang, H., Yao, N., Song, J. T., Luo, S., Lu, H., and Greenberg, J. T. (2003). Ceramides modulate programmed cell death in plants. Genes Dev. 17, 26362641. doi: 10.1101/gad.1140503

Lindermayr, C., Sell, S., Muller, B., Leister, D., and Durnera, J. (2010). Redox regulation of the NPR1-TGA1 system of Arabidopsis thaliana by nitric oxide. Plant Cell 22, 2894-2907. doi: 10.1105/tpc.109.066464

Markham, J. E., Li, J., Cahoon, E. B., and Jaworski, J. G. (2006). Separation and identification of major plant sphingolipid classes from leaves. J. Biol. Chem. 281, 22684-22694. doi: 10.1074/jbc.M604050200

Mina, J. G., Okada, Y., Wansadhipathi, N. K., Pratt, S., Shams-Eldin, H., Schwarz, R. T., et al. (2010). Functional analyses of differentially expressed isoforms of the Arabidopsis inositol phosphorylceramide synthase. Plant Mol. Biol. 73, 399-407. doi: $10.1007 / \mathrm{s} 11103-010-9626-3$

Mortimer, J. C., Yu, X., Albrecht, S., Sicilia, F., Huichalaf, M., Ampuero, D., et al. (2013). Abnormal glycosphingolipid mannosylation triggers salicylic acid-mediated responses in Arabidopsis. Plant Cell 25, 1881-1894. doi: $10.1105 /$ tpc. 113.111500

Nühse, T. S., Peck, S. C., Hirt, H., and Boller, T. (2000). Microbial elicitors induce activation and dual phosphorylation of the Arabidopsis thaliana MAPK 6. J. Biol. Chem. 275, 7521-7526. doi: 10.1074/jbc.275.11.7521

Pata, M. O., Hannun, Y. A., and Ng, C. K.-Y. (2010). Plant sphingolipids: decoding the enigma of the Sphinx. New Phytol. 185, 611-630. doi: 10.1111/j.14698137.2009.03123.x

Plett, J. M., Cvetkovska, M., Makenson, P., Xing, T., and Regan, S. (2009). Arabidopsis ethylene receptors have different roles in Fumonisin B1-induced cell death. Physiol. Mol. Plant Pathol. 74, 18-26. doi: 10.1016/j.pmpp.2009.08.004

Rivas-San Vicente, M., Larios-Zarate, G., and Plasencia, J. (2013). Disruption of sphingolipid biosynthesis in Nicotiana benthamiana activates salicylic acid-dependent responses and compromises resistance to Alternaria alternata f. sp. lycopersici. Planta 237, 121-136. doi: 10.1007/s00425-012$1758-\mathrm{z}$

Rivas-San Vicente, M., and Plasencia, J. (2011). Salicylic acid beyond defence: its role in plant growth and development. J. Exp. Bot. 62, 3321-3338. doi: 10.1093/jxb/err031

Sánchez-Rangel, D., and Plasencia, J. (2010). The role of sphinganine analog mycotoxins on the virulence of plant pathogenic fungi. Toxin Rev. 29, 73-86. doi: 10.3109/15569543.2010.515370

Saucedo-García, M., Guevara-García, A., González-Solís, A., Cruz-García, F., Vázquez-Santana, S., Markham, J. E., et al. (2011). MPK6, sphinganine and the LCB2a gene from serine palmitoyltransferase are required in the signaling pathway that mediates cell death induced by long chain bases in Arabidopsis. New Phytol. 191, 943-957. doi: 10.1111/j.1469-8137.2011. 03727.x

Serrano, M., Wang, B., Aryal, B., Garcion, C., Abou-Mansour, E., Heck, S., et al. (2013). Export of salicylic acid from the chloroplast requires the multidrug and toxin extrusion-like transporter EDS5. Plant Physiol. 162, 1815-1821. doi: 10.1104/pp.113.218156

Seyfferth, C., and Tsuda, K. (2014). Salicylic acid signal transduction: the initiation of biosynthesis, perception and transcriptional reprogramming. Front. Plant Sci. 5:697. doi: 10.3389/fpls.2014.00697

Shi, L., Bielawski, J., Mu, J., Dong, H., Teng, C., Zhang, J., et al. (2007). Involvement of sphingoid bases in mediating reactive oxygen intermediate production and programmed cell death in Arabidopsis. Cell Res. 17, 1030-1040. doi: $10.1038 /$ cr.2007.100

Simanshu, D. K., Zhai, X., Munch, D., Hofius, D., Markham, J. E., Bielawski, J., et al. (2014). Arabidopsis accelerated cell death 11, ACD11, is a ceramide-1-phosphate transfer protein and intermediary regulator of phytoceramide levels. Cell Rep. 6, 388-399. doi: 10.1016/j.celrep.2013. 12.023 
Stone, J. M., Heard, J. E., Asai, T., and Ausubel, F. M. (2000). Simulation of fungal-mediated cell death by fumonisin $\mathrm{B} 1$ and selection of fumonisin B1-resistant (fbr) Arabidopsis mutants. Plant Cell 12, 1811-1822. doi: $10.1105 /$ tpc. 12.10 .1811

Ternes, P., Feussner, K., Werner, S., Lerche, J., Iven, T., Heilmann, I., et al. (2011). Disruption of the ceramide synthase LOH1 causes spontaneous cell death in Arabidopsis thaliana. New Phytol. 192, 841-854. doi: 10.1111/j.14698137.2011.03852.x

Vlot, A. C., Dempsey, D. A., and Klessig, D. F. (2009). Salicylic acid, a multifaceted hormone to combat disease. Annu. Rev. Phytopathol. 47, 177-206. doi: 10.1146/annurev.phyto.050908.135202

Wang, D., Weaver, N. D., Kesarwani, M., and Dong, X. (2005). Induction of protein secretory pathway is required for systemic acquired resistance. Science 308, 1036-1040. doi: 10.1126/science. 1108791

Wang, E., Norred, W. P., Bacon, C. W., Riley, R. T., and Merrill, A. H. Jr. (1991). Inhibition of sphingolipid biosynthesis by fumonisins. J. Biol. Chem. $266,14486-14490$

Wang, J., Zheng, L., and Tan, R. (2007). Involvement of nitric oxide in cerebrosideinduced defense responses and taxol production in Taxus yunnanensis suspension cells. Appl. Microbiol. Biotechnol. 75, 1183-1190. doi: 10.1007/s00253-0070927-7

Wang, L., Tsuda, K., Sato, M., Cohen, J. D., Katagiri, F., and Glazebrook, J. (2009). Arabidopsis CaM binding protein CBP60g contributes to MAMP-induced SA accumulation and is involved in disease resistance against Pseudomonas syringae. PLoS Pathog. 5:e1000301. doi: 10.1371/journal.ppat.1000301
Wang, W., Yang, X., Tangchaiburana, S., Ndeh, R., Markham, J. E., Tsegaye, Y., et al. (2008). An inositolphosphorylceramide synthase is involved in regulation of plant programmed cell death associated with defense in Arabidopsis. Plant Cell 20, 3163-3179. doi: 10.1105/tpc.108. 060053

Xing, F., Li, Z., Sun, A., and Xing, D. (2013). Reactive oxygen species promote chloroplast dysfunction and salicylic acid accumulation in fumonisin B1-induced cell death. FEBS Lett. 587, 2164-2172. doi: 10.1016/j.febslet.2013.05.034

Zhang, Y., Xu, S., Ding, P.,Wang, D., Cheng, Y. T., He, J., et al. (2010). Control of salicylic acid synthesis and systemic acquired resistance by two members of a plant-specific family of transcription factors. Proc. Natl. Acad. Sci. U.S.A. 107, 18220-18225. doi: 10.1073/pnas.1005225107

Conflict of Interest Statement: The authors declare that the research was conducted in the absence of any commercial or financial relationships that could be construed as a potential conflict of interest.

Copyright (c) 2015 Sánchez-Rangel, Rivas-San Vicente, de la Torre-Hernández, Nájera-Martínez and Plasencia. This is an open-access article distributed under the terms of the Creative Commons Attribution License (CC BY). The use, distribution or reproduction in other forums is permitted, provided the original author(s) or licensor are credited and that the original publication in this journal is cited, in accordance with accepted academic practice. No use, distribution or reproduction is permitted which does not comply with these terms. 